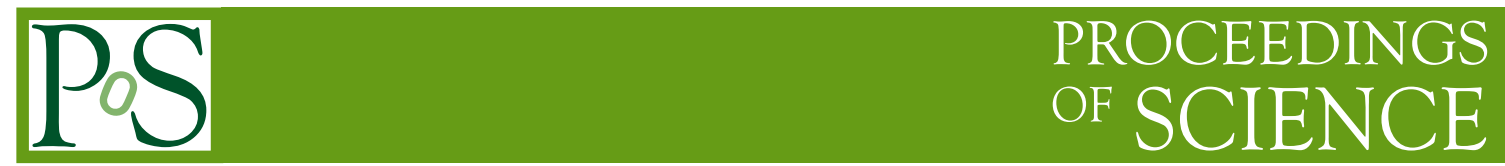

\title{
Soft QCD Measurements in ATLAS
}

\section{Paul James Laycock*}

University of Liverpool

E-mail: playcockecern.ch

\begin{abstract}
Measurements dominated by soft QCD processes, made with the ATLAS detector using LHC $p p$ data at $\sqrt{s}=0.9,2.36$ and $7 \mathrm{TeV}$, are presented. It is crucial that these processes are understood at LHC energies in order to be able to control the non-perturbative aspects of the Standard Model and allow the exploitation of the LHC data to its fullest extent. The inclusive, diffractive-enhanced and diffractive-suppressed charged particle multiplicity are compared to current models, together with track-based measurements of the underlying event.
\end{abstract}

Kruger 2010: Workshop on Discovery Physics at the LHC

December 5 - 10, 2010

Kruger National Park, Mpumalanga, South Africa

*Speaker.

†n behalf of the ATLAS collaboration. 

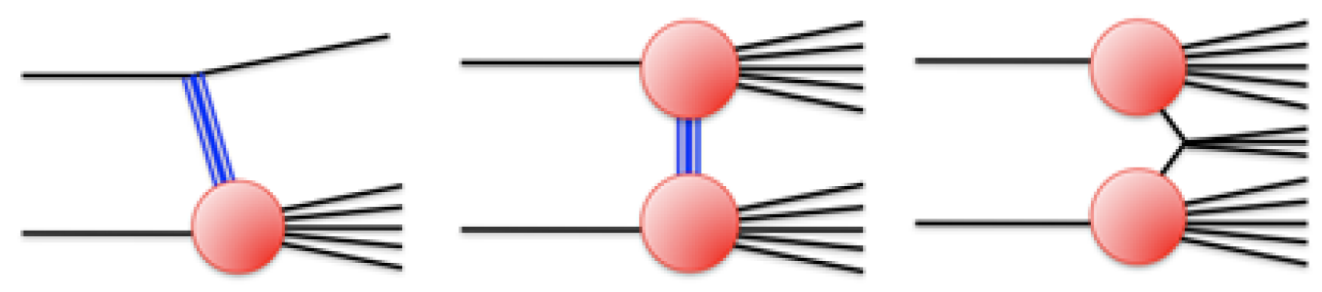

Figure 1: Contributions to the inelastic proton-proton cross section from single-diffractive, doublediffractive and non-diffractive processes.

\section{Introduction}

The Large Hadron Collider at CERN is the highest energy proton-proton collider in the world. The inclusive proton-proton cross section comprises elastic and inelastic parts, with the inelastic cross section being composed of contributions from the single-diffractive, double-diffractive and non-diffractive processes, shown in Figure 1. Models of the total inelastic cross section, while agreeing on the total rate, disagree on the size of the contributions from each of these individual processes.

The diffractive components decrease very rapidly with multiplicity and tend to be reduced to negligible levels in most physics analyses by the event selection, including e.g. vertex requirements. However, the non-diffractive component does contribute to the event samples of many physics analyses and it must therefore be understood ${ }^{1}$.

At low transverse momentum, the dominant processes arise from non-perturbative QCD and must therefore be empirically modelled. Measurements sensitive to these soft processes are crucial for testing and indeed constraining (tuning) these models, and are therefore a necessary precursor to understanding LHC collision data and proceeding with the wider physics program. Such measurements are presented here, compared with different models, and also to a tune which includes constraints from a subset of the ATLAS data.

\section{Experimental Method}

A detailed description of the ATLAS detector can be found here [1]. ATLAS employs a righthanded coordinate system with the nominal interaction point as its origin. The anti-clockwise beam direction defines the positive $z$-axis, with the polar angle $\theta$ being measured with respect to this axis; pseudo-rapidity is defined as $\eta=-\ln \tan (\theta / 2)$. The $x$-axis points towards the centre of the LHC ring, $y$ points upwards and the azimuthal angle $\phi$ in the $x-y$ plane is positive for positive $y$. The transverse component of momentum, $p_{T}$, is defined in the $x-y$ plane.

The measurements presented here primarily rely on the inner tracking detector of ATLAS, shown in Figure 2. The inner tracking detector, which is immersed in a $2 \mathrm{~T}$ axial field, consists of silicon pixel and micro-strip detectors which provide measurements of charged particle trajectories in the range $|\eta|<2.5$, complemented by a straw tube tracker in the range $|\eta|<2.0$.

\footnotetext{
${ }^{1}$ It should be noted, there is also much experimental work and theoretical interest in diffractive processes themselves.
} 

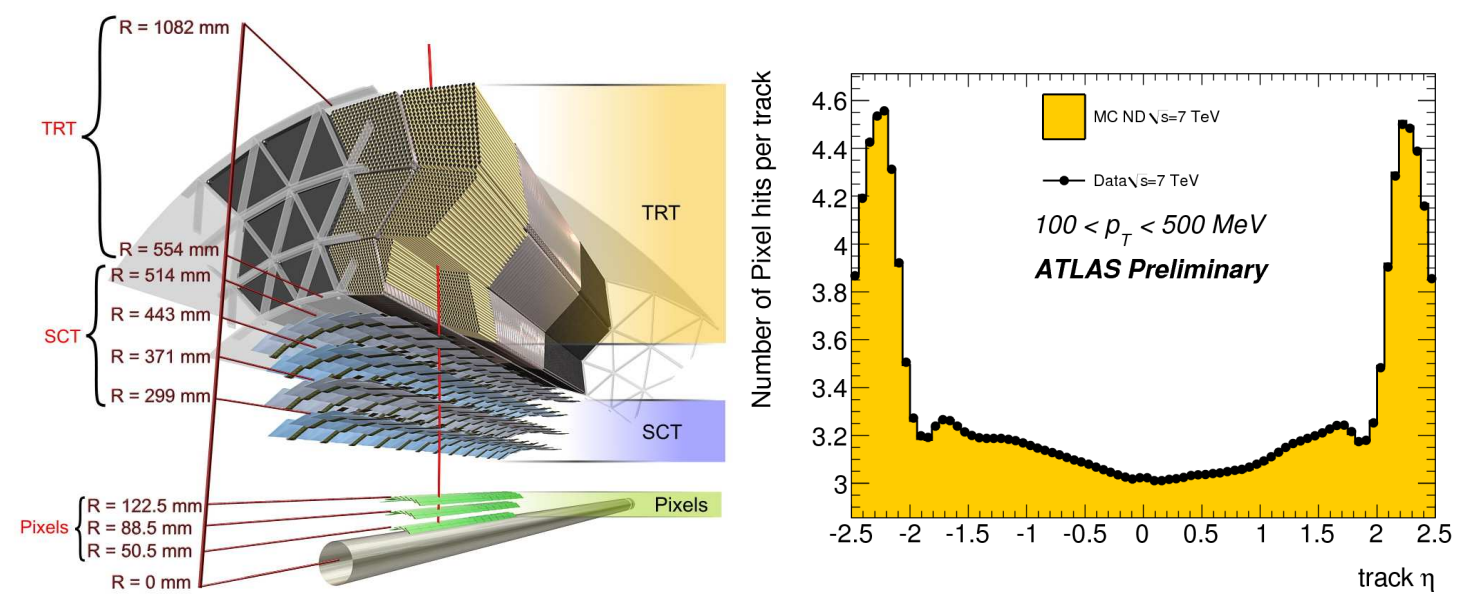

Figure 2: The ATLAS Inner Detector (left) and an example of the excellent description of this detector by Monte Carlo simulation.

The trigger for the data samples used in the analyses rely on input from the Beam Pickup Timing devices (BPTX), which are beam pick-ups attached to the beam-pipe at $z= \pm 175 \mathrm{~m}$, and the Minimum Bias Trigger Scintillators (MBTS). Each MBTS is composed of two rings $(2.09<|\eta|<$ 2.82 and $2.82<|\eta|<3.84$ ) segmented in azimuth into eight sectors and positioned at $z= \pm 3.56 \mathrm{~m}$. Trigger signals from these detectors are combined to form the MBTS trigger used in the analyses. Only data taken when the inner tracking detector was fully operational and the magnetic field was on are used. A good description of the detector by Monte Carlo (MC) simulation can be seen in Figure 2, which was used to correct the data at three centre of mass energies ${ }^{2}$.

\section{Results}

\subsection{Diffraction}

In order to study the diffractive contributions to the inelastic cross section, a sample of events with hits on only one side of the MBTS detector, single-side events, is used [2]. The data are then compared to various models, in this case PYTHIA [3] and PHOJET [4]. The ratio of single-side events to all events, $R_{S S}$, is sensitive to a combination of the single-diffractive and double-diffractive cross sections. The measured value lies in the range predicted by PYTHIA, while PHOJET significantly underestimates this value [2]. Figure 3 shows a comparison of the same models to normalised distributions, to account for the rate difference. PHOJET, while underestimating the rate, provides a better description of the data than either PYTHIA version. In terms of the diffractive cross-section, the principle difference between PYTHIA 6 and PYTHIA 8 is that the latter incorporates a model of hard diffraction. This is seen to improve the charged track multiplicity but the $p_{T}$ dependence is only described up to $p_{T} \sim 1 \mathrm{GeV}$.

\footnotetext{
${ }^{2}$ For the sake of brevity, only the results for data at a proton-proton centre of mass energy $\sqrt{s}=7 \mathrm{TeV}$ are shown here; measurements at $\sqrt{s}=0.9$ and $2.36 \mathrm{TeV}$ were also made.
} 

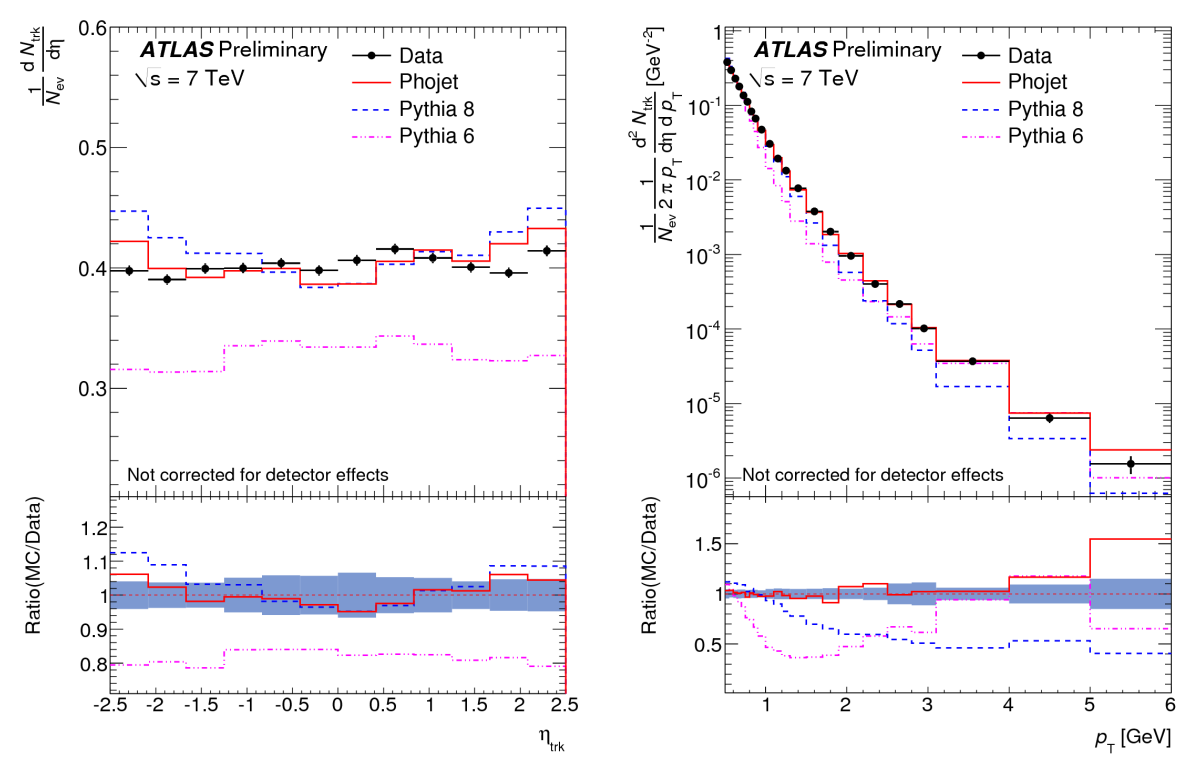

Figure 3: Normalised distributions of the charged track multiplicity as a function of $\eta$ (left) and $p_{T}$ for the diffractive enhanced sample.

\subsection{Non-diffractive measurements and MC tunes}

Minimum bias measurements can be used to tune the models in order to achieve a better description of the data. The component of primary interest is the non-diffractive cross section, thus a requirement of at least 6 tracks in the event was placed in [5] which reduces the diffractive contributions to negligible levels, allowing a comparison of data to models of the non-diffractive cross section. The results are shown in Figure 4, where the AMBT1 tune includes (among other things) input from the data shown here. While the AMBT1 tune clearly improves the description of the data, the $p_{T}$ spectrum indicates that further improvements are possible.

\subsection{Underlying Event}

Measurements of the activity in the region transverse to the hard interaction provide sensitive tests and constraints for models of the underlying event. The azimuthal direction of the leading track in the event was used to tag the direction of the hard interaction in [6]. The difference in azimuth to this leading track, $\Delta \phi$, is then calculated for every track and the transverse region is defined as $60^{\circ}<\Delta \phi<120^{\circ}$. The results are shown in Figure 5 compared to various models, with a large spread in the predictions observed.

\subsection{Extending to lower $p_{T}$}

In order to provide the best constraints to models of soft QCD, it is important to extend the data analysis to the lowest $p_{T}$ possible. In the previous analyses, the minimum $p_{T}$ for a track to be considered in the analysis was made at $500 \mathrm{MeV}$. Following improvements in both hardware understanding and software, this cut was subsequently lowered to $100 \mathrm{MeV}$ [7]. The results can be seen in Figure 6, compared to the same models as before, all of which show significant differences. 

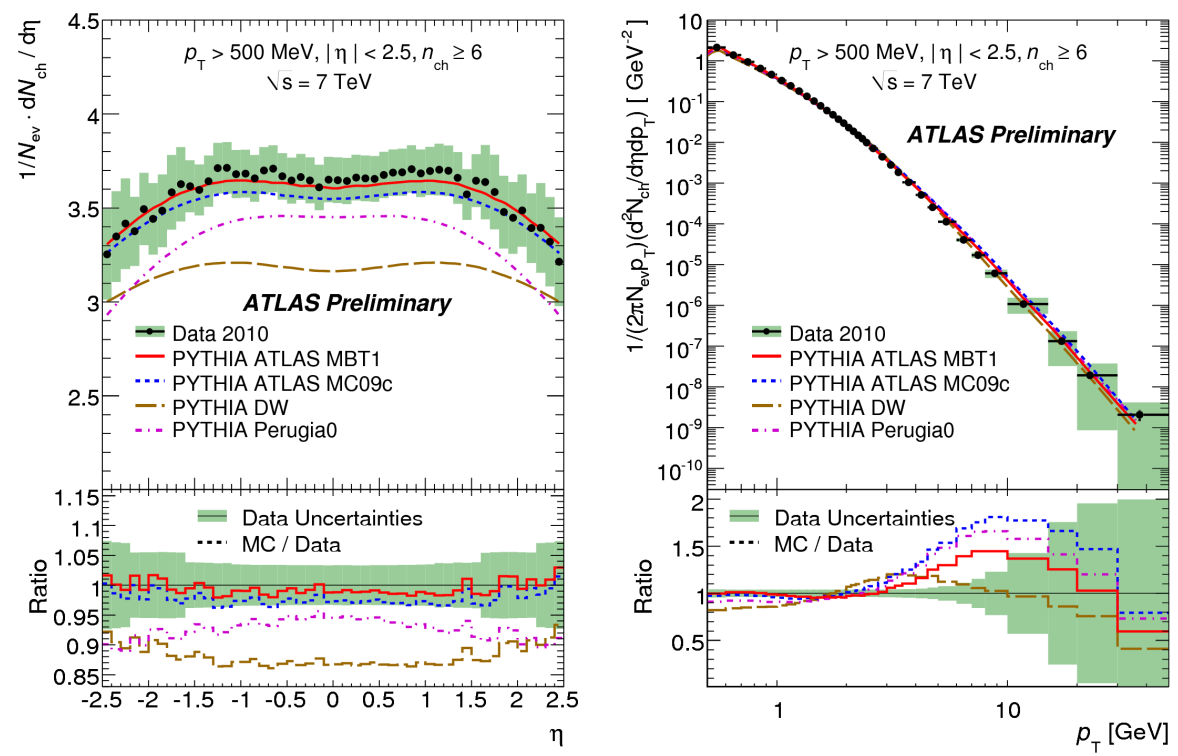

Figure 4: Normalised distributions of the charged track multiplicity as a function of $\eta$ (left) and $p_{T}$ for the non-diffractive dominated sample.
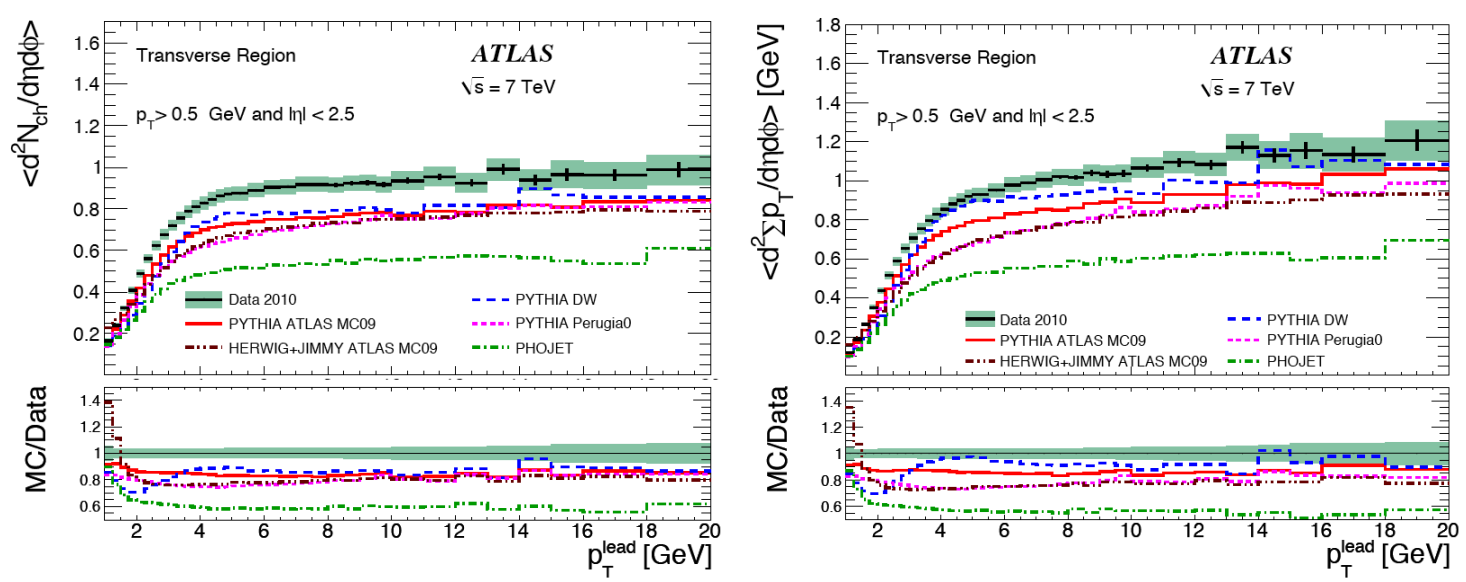

Figure 5: The number (left) and $\Sigma p_{T}$ charged particle density as a function of $p_{T}$ in the transverse region.

Despite being tuned to data at these centre of mass energies, the AMBT1 tune of PYTHIA is seen to significantly underestimate the data at this lower $p_{T}$ and the $p_{T}$ distribution itself is not described.

\section{Summary}

Measurements dominated by soft QCD processes have been made with the ATLAS detector using LHC $p p$ data at $\sqrt{s}=0.9,2.36$ and $7 \mathrm{TeV}$. The diffractive contributions to these processes are described in rate by PYTHIA, while PHOJET has a significantly better description of the differential distributions, although PYTHIA8 is significantly better than PYTHIA6. Measurements 

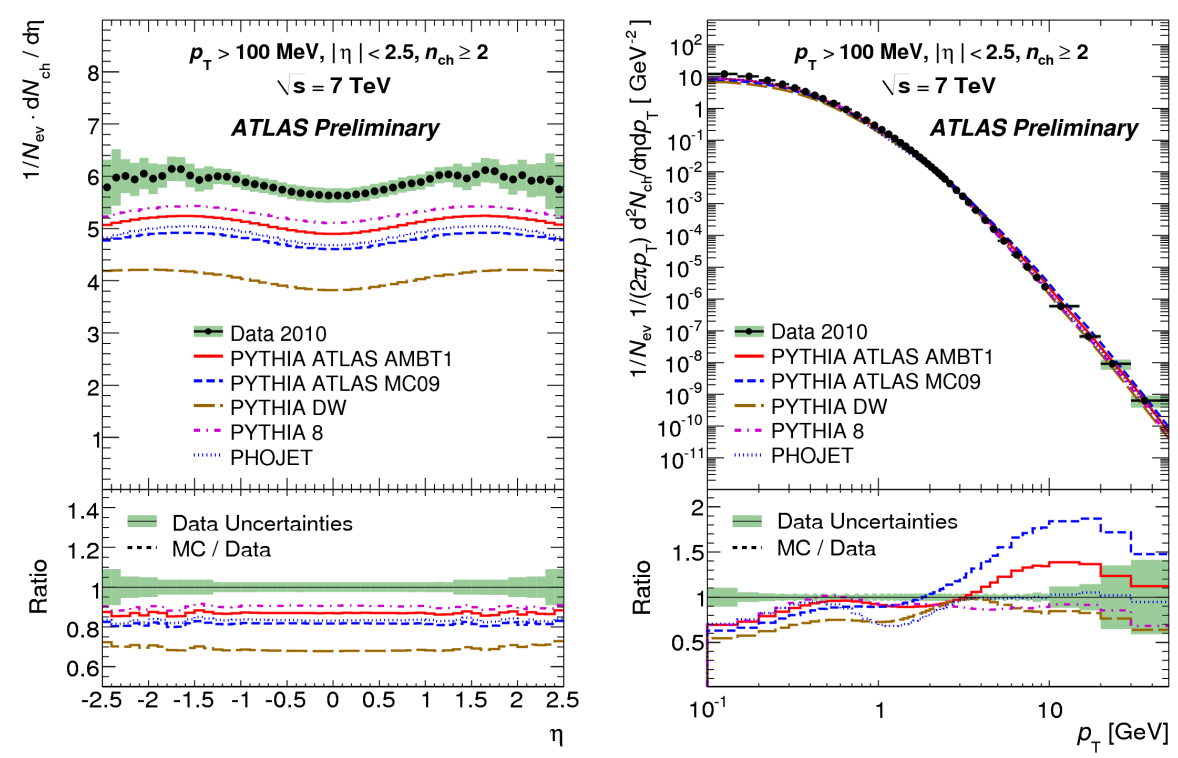

Figure 6: Normalised distributions of the charged track multiplicity as a function of $\eta$ (left) and $p_{T}$ for the non-diffractive dominated sample with lower minimum $p_{T}$.

dominated by non-diffractive events, including quantities sensitive to the underlying event, were compared to current models, revealing a large spread in the predictions. Using these data in a tune to PYTHIA resulted in a significantly improved description, as expected, although discrepancies remain. Measurements at lower $p_{T}$ show the need for further work on tuning these models, which will be crucial to exploit the LHC data to its fullest extent.

\section{References}

[1] ATLAS Collaboration. The ATLAS Experiment at the CERN Large Hadron Collider. JINST, 3:S08003, 2008.

[2] ATLAS Collaboration. Studies of diffractive enhanced minimum bias events in ATLAS. ATLAS-CONF-2010-048, 2010.

[3] Torbjorn Sjostrand, Stephen Mrenna, and Peter Z. Skands. JHEP, 0605:026, 2006.

[4] R. Engel and J. Ranft. Hadronic photon-photon interactions at high-energies. Phys.Rev., D54:4244-4262, 1996.

[5] ATLAS Collaboration. Charged particle multiplicities in $p p$ interactions at $\sqrt{s}=0.9$ and $7 \mathrm{TeV}$ in a diffractive limited phase space measured with the ATLAS detector at the LHC and a new PYTHIA6 tune. ATLAS-CONF-2010-031, 2010.

[6] ATLAS Collaboration. Measurement of underlying event characteristics using charged particles in pp collisions at $\sqrt{s}=900 \mathrm{GeV}$ and $7 \mathrm{TeV}$ with the ATLAS detector. 2010. accepted by Phys Rev D.

[7] ATLAS Collaboration. Charged particle multiplicities in $p p$ interactions for track $p_{t}>100 \mathrm{MeV}$ at $\sqrt{s}=0.9$ and $7 \mathrm{TeV}$ measured with the ATLAS detector at the LHC. ATLAS-CONF-2010-046, 2010. 